\title{
Approach to Obesity in Childhood
}

\author{
Veysel Nijat Baş ${ }^{1}$ (D) Zehra Aycan² iD \\ 1 Kütahya Health Sciences University Medical Faculty, Department of Pediatric Endocrinology, Kütahya, Turkey. \\ 2 Ankara University Medical Faculty, Department of Pediatric Endocrinology, Ankara, Turkey.
}

\begin{abstract}
Childhood obesity prevalence is increasing worldwide. The most common cause of obesity in children is a positive energy balance due to excess caloric intake in contrast to caloric expenditure combined with a genetic predisposition for weight gain. Obesity causes type 2 diabetes mellitus, hypertension, cardiovascular diseases, osteoporosis and some cancers. There is limited information on the efficacy and safety of medications for weight loss in children. In this paper, definition, etiopathogenesis, differential diagnosis, co-morbidities, and treatment of obesity in children will be reviewed.
\end{abstract}

Key words: Obesity, Childhood, Adolescence, Pediatric, Overweight.

\section{DEFINITION AND FREQUENCY}

Obesity is a chronic disease that develops with the increase in body fat mass as a result of higher amount of energy intake compared to the amount of energy spent. Obesity refers to excessive fat accumulation. Obesity is considered as one of the 10 most dangerous diseases by the World Health Organization, and it may affect all endocrine, metabolic, cardiovascular and respiratory systems of the body and cause a wide variety of problems and death (14).

Childhood obesity is a rising global health problem. The number of overweight and obese children in the 0-4 age group increased from 32 million in 1990 to 41 million in 2016 throughout the world. The number of overweight and obese children in the world is expected to reach 70 million by 2025 , if the current rate of increase in the frequency of obesity continues (4-8). In COSI TUR 2016 research study conducted in 67 provinces representing Turkey, when body mass index (BMI) of 2nd grade elementary school children were assessed according to Z-Score, 9.9\% were found to be obese and $14.6 \%$ were found to be overweight; however, in the previous COSI 2013 research, obesity was determined as $8.3 \%$ and the percentage of overweight children was determined to be $14.2 \%$. The rate of obese and overweight children are observed to increase in the more recent study compared to COSI 2013 report (9). There is still no specific treatment strategy in developed countries despite the serious increase in the obesity rate in childhood in the last 30 years and most of the performed studies are directed towards prevention of childhood obesity (2-6). 


\section{ETIOPATHOGENESIS}

Epigenetics is a term that refers to the hereditary rearrangement of gene expression without alteration in the base sequence of DNA. Epigenetics is thought to play a major role in the serious increase in obesity during the past 30 years. Children with obese parents are at high risk for obesity. If one of the parents is obese, then the risk is $30 \%$, however if both of the parents are obese, then the risk of obesity for the child is $90 \%$ (1-5,10-12). In addition, excessive weight gain during pregnancy, tobacco use, gestational diabetes mellitus increases the risk of obesity in children. Toxins, drugs, infections, and exogenous hormones are considered to be other conditions that result in obesogenic epigenetic changes. Exposure to environmental endocrine disrupting chemicals such as pesticide dichlorodiphenyl-trichloroethane or bisphenol A were found to increase the likelihood of triggering or aggravating obesity in some epidemiological studies. Though genetic factors play a role in a person's susceptibility to obesity, an obesogenic environment must be for present for phenotypic expression (3-7,10-12).

Various environmental factors contribute to the development of obesity. Sedentary lifestyle due to industrialization is the biggest threat in the formation of obesity. Sedentary lifestyle despite excessive and fast eating are the most important reasons for obesity in children. The presence of environmental factors such as increased intake of foods with high glycemic index, sugarcontaining drinks, corn starch foods and beverages, large portions of ready-made foods, fast food service, reduced habit of eating with the family, decreased physical activity, shortened sleep time and scarcity of playgrounds contribute to the development of obesity. Duration of watching television is one of the most environmentally related effects on the development of obesity in childhood. Playing electronic games has also been associated with obesity in childhood with weaker effect on obesity compared to watching television. This has been attributed to the fact that electronic games do not contain food ads $(2-7,13)$.

In recent years, intestinal microbiota has been an important focus in the investigation of the obesogenic environment. Many studies have shown that obesity and its complications are associated with changes in intestinal microbiota. Some researchers have suggested that there is a relationship between the bacteria settled in the intestinal tract with the potential for weight gain. Animal studies have shown that administration of antibiotics early in life increases the susceptibility to obesity. Although many observational studies and meta-analyses support this possibility, it has been suggested that the magnitude of this effect may be minor or even clinically insignificant. A relationship between prenatal antibiotic exposure and obesity in early childhood was reported. In another extensive study in which a relationship between antibiotic exposure in early infancy and subsequent weight gain could not be found, exact opposite results were observed. Whether these mechanisms are related to human obesity, or not, and if this is the case, whether they significantly contribute to obesity epidemic, or not remains uncertain (5-8,10-14)

\section{DIAGNOSIS}

Weight assessment in children with obesity is made according to the child's age and the severity of obesity. The goal of measurement methods is to determine the ratio of adipose tissue and lean tissue. Direct and indirect methods are used to measure body fat. Body mass index (BMI) is a commonly used indirect method recommend by WHO in field studies related to clinical obesity, rather than direct fat measurements such as X-ray absorptiometry, wholebody magnetic resonance imaging, and bioelectrical impedance analysis, which are expensive, laborious and difficult methods for measuring fat mass. Body mass index is the most practical and reliable method used in defining obesity $(2-8,15-17)$. BMI is not used in children up to 2 years old. Instead, the weight percentile of the children is compared with the height percentile. Children who have a higher increase in weight percentage than height percentage should be closely monitored. BMI charts are used for children between the ages of 2-20. Specific percentile curves have been created for our country starting with infancy. BMI's of $85-94 \%$ (> 1.5SD) percentile indicates overweight, and BMI being over 95\% (> 2SD) indicates obesity according to age and gender between the ages of 2-20. In normal children, there is a " $\mathrm{J}$ " shaped curve in both charts, since BMI decreases between the years 2-6 and increases between the years 6-20 (3-7). The initial decline and subsequent increase in BMI is called the "adiposity rebound". If a child does not have a decrease in $\mathrm{BMI}$ or has an early rise between the ages of $2-6$, then that child is at risk for obesity. Today, quiet practical calculation methods have been developed with the utilization of technology (15-17). BMI SD and percentile calculations 
according to the height and weight standards of children of our country can be easily calculated with a program developed by the Association of Child Endocrinology and Diabetes. It is possible to access the program called ÇEDD-Oksoloji / ÇEDD Çözüm via smart phones (https://www. https://www.ceddcozum.com/Home/ Change?LanguageAbbreviation=tr). It is an easy-to-use practical program and can be used to define obesity in children and to evaluate their height percentiles.

\section{DIFFERENTIAL DIAGNOSIS}

Childhood obesity is generally exogenous (primary) type. There is no underlying pathology for this type. Exogenous obesity is basically caused by the fact that the calorie intake is more than the energy spent and therefore it is a preventable situation. Causes such as age, gender, genetics, nutritional habits, socioeconomic status and lack of physical activity plays role in the development of this condition. Secondary type obesity, having an incidence of less than $1 \%$ in childhood, develops due to another underlying pathology (genetic syndromes, endocrine or other causes) (4-8).

Taking a detailed history is very important to reveal the causes of secondary obesity in the approach to obesity disease. Family history, prenatal and postpartum history, postpartum medical care history should be taken in detail. Diet history should be questioned; breastfeeding time, bottle feeding, transition to supplementary foods should be noted. The activities of the child should be questioned; exercises or daily activities, sleep time and duration, as well as obesity-related comorbid diseases should be investigated, history of known diseases and drug use should be questioned. The psychological health of the child should also be questioned and it should be understood whether the onset of obesity is related to intense stress (traffic accident, loss of parents or friends, etc.), or not. Increased eating behavior may be associated with feelings of pleasure and stress relief $(1,3,6-8)$.

Differential diagnosis in children with obesity begins with the evaluation of linear growth. Accelerated linear growth is observed in children with exogenous obesity. These children are at risk for the early development of their secondary sexual characteristics and their bone age may also be more than their chronological age. Children with obesity due to an underlying endocrinopathy have decreased linear growth. Acquired hypothyroidism, cortisol excess (corticosteroid therapy,
Cushing Syndrome), growth hormone deficiency, and pseudohypoparathyroidism are examples of endocrine disorders causing childhood obesity. These disorders are usually associated with overweight or mild obesity rather than severe obesity. Endocrine causes were found in few of the obese children who were enrolled in many retrospective studies. Routine laboratory examinations directed to endocrine causes are not recommended if the patient's height is not shorter than his/her genetic potential and/or growth rate has not decreased according to age and gender $(1,4,5,7,8)$.

Monogenic obesity should be considered in children who gained excess weight in early infancy, especially in children with severe obesity before the age of 5 . These children may develop developmental delay, short height, dysmorphic face, and hyperphagia. Though Prader Willi, BardetBiedl, Fragile-X, Albright hereditary osteodystrophy, Alström syndrome, congenital leptin deficiency, proopiomelanocortin (POMC) deficiency, MC4R deficiency and Cohen's syndrome are obesity-related syndromes, other genetic causes that are not associated with these syndromes might also be present. Syndromic and genetic causes should come to mind at first in the presence of short stature, mental retardation, bone age retardation, and findings of pathological physical examination (1,4-7).

\section{PHYSICAL EXAMINATION}

Physical examination is both important and difficult in children with obesity. In particular, adolescent patients may wear loose clothing or tight corsets to hide their obesity thus the clinician should be careful about this. Moreover, increased adipose tissue or increased hair growth are other reasons that make physical examination difficult. It is not always easy to determine early thelarche in girls or gynecomastia in boys $(4,7,8)$. Attention should be paid to skeletal system problems in children with obesity. Children with curvature in their legs should be evaluated in terms of Blount Disease (tibia vara) and additional radiological examinations should be requested. Although scoliosis is more common in obese children than in children with normal weight, its diagnosis is more difficult due to excessive fat. The clinician should keep the possible skeletal problems in mind and request further radiological examination when necessary. In addition, physical examination should be extended according to the patient's additional complaints such as neck circumference, and tonsils should be examined in a patient suffering from snoring (3-8), 
Relationships between obesity and a wide range of health problems such as cardiovascular diseases, metabolic diseases, respiratory system diseases, chronic diseases (hypertension and diabetes), dyslipidemia, sleep apnea syndrome, asthma, polycystic ovarian syndrome, depression and quality of life deterioration, non-alcoholic liver steatosis, femoral head epiphysis shift have been found in previous studies (3-7).

\section{OBESITY COMORBIDITIES}

\section{Hypertension}

It is more common in obese children than in children with normal weight. Activation of the sympathetic system, increased sodium reabsorption, hyperinsulinemia, activation of renin angiotensin aldosterone are factors facilitating hypertension (HT) in obesity. Three separate measurements should be made at least 1 week apart for the diagnosis of HT. Values for the diagnosis of HT differ by age in children. It should be performed by evaluating normal levels according to age, gender and height in children under 13 years of age. Blood pressure is ordinarily lower at night. Blood pressure measured at night should normally show a reduction of up to $10 \%$. Disruption of this circadian rhythm in childhood obesity is considered as an early sign of hypertension. In children diagnosed with stage $2 \mathrm{HT}$, final organ damage should be evaluated. In particular, kidney function tests should be requested. Primarily a lifestyle change and diet should be tried for treatment. Low sodium is recommended for diet. Although weight loss constitutes the basis of HT treatment in obese children, pharmacotherapy should be initiated in patients with persistent HT or in patients with end organ damage $(3,6,18)$.

\section{Dyslipidemia}

This disease is more common in obese children, characterized by high triglyceride and low HDL levels. Dyslipidemia associated with obesity responds very quickly to weight loss. Further research should be applied and primary dyslipidemia should be investigated in cases of dyslipidemia that do not respond to weight loss or manifest itself with different values than high triglyceride and low HDL levels $(4,7,19)$.

\section{Sleeping Disorders}

Obese children may experience sleep disturbances such as snoring, sleep apnea syndrome, daytime sleepiness, hyperactivity, depression, and nocturnal enuresis with new onset. It is recommended that the clinician should be careful about this issue and make necessary clinical evaluations by questioning the sleep pattern $(7,8)$.

\section{Glucose Metabolism Disorders}

Continued excessive energy intake leads to impaired glucose metabolism and insulin resistance in obese individuals. Insulin resistance is associated with obesityrelated comorbidities and cardiometabolic risk. Insulin sensitivity is usually evaluated by hyperinsulinemic euglycemic clamp tests or frequent blood sampling for plasma insulin and glucose concentrations with intravenous glucose infusion. However, these techniques are used rarely in daily practice. Evaluation of insulin sensitivity based on fasting or post-meal glucose and insulin concentrations is clinically performed. These are HOMA-IR (homeostasis model of insulin resistance), QUICK index, and Matsuda index. HOMA-IR shows a high correlation with fasting insulin values in children and it is seen as a more suitable method to show insulin resistance compared to fasting insulin measurement alone. HOMA-IR normal values vary in different societies and age ranges. In a study by Kurtoğlu et al. insulin resistance in obese children was evaluated, the limit levels for HOMA-IR were calculated as 2.67 for prepubertal boys, 2.22 for prepubertal girls and 5.22 for pubertal boys, 3.82 for pubertal girls in our country (20-24).

Serum glucose screening is also recommended in obese children. While impaired fasting glucose is expressed as FBG 100-126 mg / dl in repeated measurements, impaired glucose tolerance means that BG is $140-199 \mathrm{mg} / \mathrm{dl}$ at OGTT at the 2nd hour. These disorders may be associated with type $2 \mathrm{DM}$. However, $\mathrm{HbA} 1 \mathrm{C}>5.9 \%$ was suggested to be associated with insulin secretion, insulin sensitivity and $B$ cell dysfunction $(1,4,8)$.

\section{Treatment}

Necessary measures to prevent obesity should be taken at a very early period. Breastfeeding alone in the first 6 months and co-administration of supplementary foods after 6 months with should be recommended to parents who are in charge of taking care of the child in all child follow-up outpatient clinics. Fruit juice, sweet drinks and carbohydrate loaded formulas should be avoided after switching to supplementary foods. The family should be 
involved in the nutritional management, the diet should be rich in vegetables and fruits, and foods containing carbohydrates with high glycemic index should be limited. Physical activity is another aspect that is as important as nutrition. Physical activity should be suitable for the child's age and should complement the daily nutrition plan. Active lifestyle and limitation of television and video games during the day is very important for the prevention of obesity $(3,4,7,8)$.

Pharmacological treatment is very limited in the treatment of obesity in children and adolescents. Orlistat is the only drug approved by the FDA for the treatment of obesity in adolescents (> 12 years). Orlistat, a lipase inhibitor, prevents the absorption of approximately one-third of the fat taken in a meal. The recommended orlistat dosage is $120 \mathrm{mg} 3$ times a day with meals. Its efficacy was found to be limited in placebo-controlled studies. Diarrhea, abdominal pain, bloating and fatty stool are among the side effects that limit the use of orlistat. It is recommended to take a multivitamin when using orlistat, since it prevents the absorption of fat-soluble vitamins. Phentermine is another FDA-approved drug for short-term treatment (12 weeks) over the age of 16 years. There are few studies on phentermine which is an asympathomimetic amine derivative. Blood pressure and heart rate should also be evaluated during screening and monitoring for heart disease before use. Topiramate and glucagon-like peptide- 1 analogs are found among other drugs that have not been approved for the treatment of obesity in children. Studies on these pharmacotherapies were uncontrolled and the number of cases were insufficient, more experience seems to be necessary to understand the efficacy of these medications in obesity treatment $(1,3,7,8)$.

One of the most frequently used drugs in clinical practice is metformin though it is not approved by the FDA in treatment of obesity. Metformin is approved for Type 2 DM. Its short-term use can be recommended with expert opinion and is frequently used by pediatric endocrine specialists if obesity is accompanied by acanthosis nigricans, glucose metabolism disorders and other comorbidities such as polycystic ovary syndrome (PCOS). Metformin is a guanidine derivative used in child and adult Type 2 DM. It reduces fasting hyperinsulinemia and prevents Type $2 \mathrm{DM}$, and supports weight loss in some obese individuals by increasing hepatic and muscle insulin sensitivity. It is contraindicated to use in cases such as kidney failure, congestive heart failure, lung failure and liver failure. Nutrition and lifestyle changes should be tried at first for type 2 DM treatment. Pharmacologically, metformin having FDA approval can be used in children over the age of 10 . The first treatment option should include lifestyle changes where healthy nutrition and physical activity are enhanced whether insulin resistance is present in obesity, or not. Exercise increases hepatic glucose uptake and glycogen synthesis and reduces hepatic glucose production, thereby lowers fasting glucose and insulin levels $(3,8,24,25)$.

Obtaining positive results after bariatric surgery procedures for severe obesity in adolescents similar to adults has increased the popularity of this method in recent years. Roux-en- $Y$ gastric bypass and sleeve gastrectomy are the methods that are generally used in these age groups. Insufficient number of patients and follow-up duration, and lack of standardization of the procedures may be considered the limitations of the studies on this subject. This method, which is not a part of the routine treatment, can be applied in selected cases, with the decision of a multidisciplinary team experienced in adolescents with severe obesity and comorbid diseases, who cannot lose weight despite drug treatment $(3,6,7,26)$.

\section{CONCLUSION}

Childhood obesity is a preventable condition that also reflects into adulthood and causes important problems. Obesity is an important disease causing type 2 diabetes mellitus, hypertension, cardiovascular diseases, osteoporosis and some types of cancer. In addition, obesity adversely affects a person's social life, reduces self-esteem and can cause mental problems. It is important to recognize these children as early as possible to investigate and prevent possible pathologies. The goal of managing a child with obesity should be not only to reduce BMI, but also to prevent complications and reduce existing comorbidities. It is difficult to treat when obesity and associated comorbid conditions develop, therefore primary prevention appears to be more effective in these situations. The lack of sufficient evidence-based research on the management of obesity in children prevents the application of analytical clinics. The development of the pediatric obesity algorithm will enable it to be used by experts as a comprehensive evidence-based road map for the diagnosis and management of children with obesity. 


\section{Declarations}

The authors received no financial support for the research and/or authorship of this article. There is no conflict of interest.

\section{REFERENCES}

1. Styne DM, Arslanian SA, Connor EL, Farooqi IS, Murad MH, Silverstein JH, et al. Pediatric obesity-assessment, treatment, and prevention: an endocrine society clinical practice guideline. J Clin EndocrinolMetab. 2017 Mar;102(3):709-57.

2. World Health Organization. Obesity and overweight. Available at: https: / / www.who.int/ news-room/ fact-sheets / detail/ obesity-andoverweight/ (Accessed on 1 June 2020).

3. Bereket A, Atay Z. Current status on obesity in childhood and adolescence; prevalence, etiology, co-morbidities and management. Obesity Medicine. 2016 May; (3):1-9.

4. Boland CL, Harris JB, Harris KB. Pharmacological management of obesity in pediatric patients. Ann Pharmacother. 2015 Feb;49(2):22032.

5. Brown T, Moore TH, Hooper L, Gao Y, Zayegh A, Ijaz S et al. Interventions for preventing obesity in children. Cochrane Database Syst Rev 2019;7(7):CD001871. Published 2019 Jul 23. doi:10.1002/14651858.CD001871.pub4

6. Simmonds M, Llewellyn A, Owen CG, Woolacott N. Simple tests for the diagnosis of childhood obesity: a systematic review and metaanalysis. Obes Rev. 2016 Dec;17(12):1301-15.

7. Styne DM, Arslanian SA, Connor EL, Farooqi IS, Murad MH, Silverstein JH et al. Pediatric Obesity-Assessment, Treatment, and Prevention: An Endocrine Society Clinical Practice Guideline. J Clin Endocrinol Metab. 2017 Mar 1;102(3):709-57

8. Sabin MA, Kiess W. Childhood obesity: Current and novel approaches. Best Pract Res Clin Endocrinol Metab. 2015 Jun;29(3):327-38.

9. https://hsgm.saglik.gov.tr/depo/haberler/turkiye-cocukluk-cagisismanlik/COSI-TUR-2016-Kitap.pdf (Accessed: 20.05.2020)

10. Warner M, Wesselink A, Harley KG, Bradman A, Kogut K, Eskenazi B. Prenatal exposure to dichlorodiphenyltrichloroethane and obesity at 9 years of age in the CHAMACOS study cohort. Am J Epidemiol 2014 Jun 1;179(11):1312-22.

11. Vom Saal FS, Nagel SC, Coe BL, Angle BM, Taylor JA. The estrogenic endocrine disrupting chemical bisphenol A (BPA) and obesity. Mol Cell Endocrinol 2012 May 6;354(1-2):74-84

12. Dugas C, Perron J, Kearney M, Mercier R, Tchernof A, Marc I, et al. Postnatal prevention of childhood obesity in offspring prenatally exposed to gestational diabetes mellitus: where are we now? Obes Facts 2017 Aug;10(4):396-406

13. Gilbert-Diamond D, Li Z, Adachi-Mejia AM, McClure AC, Sargent JD. Association of a television in the bedroom with increased adiposity gain in a nationally representative sample of children and adolescents. JAMA Pediatr 2014 May;168(5):427-34.

14. Paolella G, Vajro P. Childhood obesity, breastfeeding, intestinal microbiota, and early exposure to antibiotics. JAMA Pediatr. 2016 Aug $1 ; 170(8): 735-7$.
15. Reilly JJ, Armstrong J, Dorosty AR, Emmett PM, Ness A, Rogers I et al; Avon longitudinal study of parents and children study team. Early life risk factors for obesity in childhood: cohort study. BMJ. 2005 Jun 11;330(7504):1357.

16. Cunnigham SA, Kramer MR, Narayan KM. Incidence of childhood obesity in United States. N Engl J Med. 2014 Jan 30;370(5):403-11.

17. McCuen-Wurst C, Ruggieri M, Allison KC. Disordered eating and obesity: associations between binge-eating disorder, night-eating syndrome, and weight-related comorbidities. Ann N Y Acad Sci. 2018 Jan;1411(1):96-105.

18. Faulkner B. Monitoring and management of hypertension with obesity in adolescents. Integ Blood Pressure Control. 2017 Nov 20;10:33-39.

19. Cook S, Kavey RE. Dyslipidemia and pediatric obesity. Pediatr Clin North Am. 2011 Dec;58(6):1363-73

20. Haemer MA, Grow HM, Fernandez C, Lukasiewica GJ, Rhodes ET, Shaffer LA, et al. Addressing prediabetes in childhood obesity treatment programs: support from research and current practice. Child Obes. 2014 Aug;10(4):292-303.

21. Elder DA, Hornung LN, Herbers PM, Prigeon R, WooJG, D'Allessio DA. Rapid deterioration of insulin secretion in obese adolescents preceding the onset of type 2 diabetes. J Pediatr. 2015 Mar;166(3):672-8.

22. Reinehr T. Metabolic Syndrome in Children and Adolescents: a Critical Approach Considering the Interaction between Pubertal Stage and Insulin Resistance. Curr Diab Rep. 2016 Jan;16(1):8.

23. Kurtoğlu S, Hatipoğlu N, Mazıcıoğlu M, Kendirici M, Keskin M, Kondolot M. Insulin resistance in obese children and adolescents: HOMA-IR cut-off levels in the prepubertal and pubertal periods. J Clin Res Pediatr Endocrinol. 2010 Aug;2(3):100-106.

24. GBD 2015 Obesity Collaborators, Afshin A, Forouzanfar MH, Reitsma MB, Sur P, Estep K, Lee A et al. Health Effects of Overweight and Obesity in 195 Countries over 25 Years. N Engl J Med 2017 Jul 6;377(1):13-27.

25. McDonagh MS, Selph S, Ozpinar A, Foley C. Systematic review of the benefits and risks of metformin in treating obesity in children aged 18 years and younger. JAMA Pediatr. 2014 Feb;168(2):178-184.

26. Paulus GF, de Vaan LE, Verdam FJ, Bouvy ND, Ambergen TA, van Heurn LW. Bariatric surgery in morbidly obese adolescents: a systematic review and meta-analysis. Obes Surg. 2015 May;25(5):86078 . 